former, and, as you see, you are enabled to keep down the heel, and to turn outwards the foot still more. It is comparatively seldom that I use the c-splint, the others being usually sufficient for a perfect result. A good boot for subsequent wear, which may be put on at home without the assistance of the surgeon, is still a desideratum. I have not seen one which satisfies me.

Paralytic talipes is to be treated according to the same principles. You will here find, however, unless the case be of old standing, that you may generally get the foot into position without the aid of tenotomy. In any case, the only structure likely to require division is the tendo Achillis. I show you this girl of twelve, who suffered from this form of talipes. She had been treated for many months before we saw her with an apparatus, but could still only walk on the ball of the toes. The tendo Achillis was remarkably tense, and the foot could not be pressed into position. We therefore divided the tendon, and, as you see, she can now place the foot flat on the floor, and walks perfectly well, except as regards the want of power in the peronei. You will sometimes hear of galvanism as a suitable remedy for these cases. It is not so. It is only useful in preventing the occurrence of the deformity. When the case comes under the surgeon as one of talipes, the time for using galvanism has lnng since passed away. Therefore do not dream of it.

I think I have said enough of treatment to enable you to appreciate the principles and details of our practice; and I will leave the consideration of exceptional varieties of the affection till opportunities of observing them occur.

\section{INFLUENCE OF SOLENOIDS ON THE NERVOUS SYSTEM.}

By GEORGE SIGERSON, M.D., Ch.M., F.L.S.,

Member of the Royal Irish Academy, of the Scientific Society of Brussels, and of the Clinical and Anthropological Societies of Paris.

IN a recent contribution to the study of certain phenomena connected with hystero-epilepsy and cerebral anæesthesia, ${ }^{*}$ I proceeded (after showing that their appearance depended, in given cases, on physical causes) to indicate what might be the efficient agents. The conclusion arrived at from an analysis of the various experiments was that, generically speaking, a peripheral stimulus was required which, by affecting the superficial nerve-fibres, should by that medium react upon the nervecentres. Several kinds of stimuli had proved effectual, such as the galvanic and faradaic currents, the influence of solenoids and magnets at a distance. The application of metal discs, having been found to give rise to feeble but efficient currents, came under the same head. The generic term was adopted in order to include stimuli other than electrical, such as, for instance, the irritation of a sinapism, a ligature, or (possibly) the actual cautery. On the other hand, I ventured to point out that, before excluding the possibility of physical agency in cases where apparently inefficient objects were successfully applied, it was desirable to investigate whether some unnoticed cause had not been at work to disturb the electrical condition of the part. Thermo-electric currents, for instance, might be produced by inequalities of temperature not generally suspected of possessing such an influence; and other readily overlooked agencies might give rise to electro-motive action, sufficient to furnish the physical stimulus required.

If I refer to this subject at present, it is because recent researches have come to corroborate these propositions, and to extend our knowledge of a question which is both interesting and important, whether we consider it from a pathological or a physiological standing-ground. Obviously, however clear the deductions drawn from experiments on the human patient might be, it still remained desirable that these should be verified and controlled by investigations on animals in the physiological laboratory, above all when the agency of expectant attention had been so much insisted on. Since his experiments at La Salpêtrière, which I have described, Professor Schiff has devoted his attention to this point. Having first made certain that the alleged phenomena were real, he reasoned that, as it was impossible to suppose the existence of special laws for hysterical cases, the phenomena in question must be the expression of a general property of the animal machine, which becomes visible when the nature of the disease produces or fosters a great excitability of the nervous system. Moved by such considerations, Professor Schiff undertook a series of experiments which have given some remarkable results. $t$ They were conducted chiefly with a view

An Examination into certain recently reported Phenomena in connection with Hystero-Epilepsy and Cerebral Anæsthesia, Pritish MEDICAL JovRnaI., February

Hystero- Epilepsy
ist and 8 th, 1879 .

+ Contributions à l'Étude des Effets des Pobines d'Induction sur le Système Nerveux. Par M. Schiff, Archives des Sciences Phy's. ct Nat., Génève, IE 79 . to determine what influence, if any, was possessed by solenoids and magnets; but some observations were also made with respect to the influence of metallic discs. The results may be briefly summarised as follows :

Metallic Discs. - It has been already indicated in these columns that Dr. Vierordt of Tiibingen found that, on the application of a metallic disc to the abdomen of the frog, reflex sensibility became augmented in the posterior extremities. Professor Schiff states that he has been a witness to the fact that when metallic discs, from one and a half to two centimitres in length, were applied to the posterior extremities, after destruction of the nerve-centres, electric currents of some energy resulted. The principal current passed through the metal, and the derived currents through the animal membrane. Hence they could provoke, in miniature, all the effects known to flow from what has been called electrotonus of nerves ; in other words, they could, according to their direction, augment or diminish, or generally modify nervous excitability. In the case of the human subject, the application of metallic discs to the surface of the hand gives derived currents in the galvanometer which are much weaker than those of the frog. Their intensity may be modified by the nature of the metal, the moist condition and difference of temperature presented by the different parts of the skin to which the application is made. It is regarded as possible that the unequal pressure of different parts of the disc upon the skin may give rise to currents.

Professor Schiff had supposed at first, with others, that the metallic discs became active because currents were developed by contact with the different secretions of the skin. In order to test whether this were so, he interposed between an amalgamated zinc disc and the skin a layer of blotting-paper, kept moist with a concentrated solution of sulphate of zinc. Notwithstanding this, the usual phenomena took place. Hence he concludes that it is scarcely probable that chemical differences produce the currents; and, after these experiments, he prefers to suppose the existence of a thermo-electric current, such as I have suggested.

In the experiments on animals, the extremity, when placed within the cavity of the solenoid, was carefully isolated by the interposition of a glass cylinder, or wood covered with wax, in the case of frogs. When the extremities of mammals (dogs) were made use of, it was found that the body of the concentric bobbin and the hair of the animal sufficed to secure isolation. Sometimes, in the case of frogs, the pole of a strong magnet, or electro-magnet, was employed instead of the bobbin, and placed at a distance of four millimetres from the nerve or extremity. The experiments are divisible into two series: (I) those which relate to the peripheral nerves, and (2) those which concern the nervecentres.

I. Peripheral Experiments.-In the first series of investigations, frogs only were made use of. An extremity was taken, or else the whole frog, with the exception of the brain and upper part of its spinal marrow, which had been removed. The results were negative. It is to be remarked that, in Dr. Vierordt's experiments, the upper portion of the spinal cord was not removed, though the cerebral lobes were taken away. Professor Schiff is far from considering that the negative results of these experiments prove the nullity of the agencies in question; on the contrary, he hopes, by improving his methods of research, to obtain positive results.

Here it is necessary to point out that these negative results do not at all affect the authenticity of the phenomena observed in the human subject. In such a case, the hemianrsthesia is complete; in other words, one entire half of the organism is affected, externally at least, by insensibility. Hence it is imperative on us to conclude that the intracranial nerve-masses are involved in some way, and, therefore, between a patient thus affected and an animal from which the brain and upper part of spinal cord have been removed, the conditions are not analogous.

2. Central Experiments.-As subjects for the second series of researches, live dogs were chosen. It should be premised that Professor Schiff found, in 1871 , that those parts of the brain which MM. Hitzig and Fritsch regarded as motor centres, have no direct influence on motion, but are really centres of tactual sensibility for various portions of the body. No motor paralysis results from their destruction, but tactual sensibility is abolished, and with it the sense of position as regards the extremities affected. The movements which irritation of those portions of the cortex produces are to be considered as movements due to reflex sensibility, and the disorderly state of some movements, after extirpation of the aforesaid parts, should be looked upon as the result of the absence of tactual sensibility, the organ of which has been abolished. Faithful to the principles of the experimental school, Professor Schiff, whilst rejecting the doctrine of the existence of motor centres in animals, admits their presence in man, because clinical observation, and especially the important investigations of Pro- 
fessor Charcot, appear to have demonstrated their existence beyond all question. For some time, he was disposed to admit, after Professor Ferrier's experiments, that such centres probably existed also in monkeys; but he considers that the experiments of Luciani and Tamburini (notwithstanding their personal inferences) tend to prove the contrary. Most physiologists, especially in Germany, he adds, have rallied to this opinion. Even Professor Hitzig admits that, in those centres, which he still calls "motor", he recognises centres of the sense of muscular attitude. Lastly, Professor Schiff's opinion has been completely confirmed by the fine experiments of Dr. Munk. According to the investigations of Drs. Goltz and Munk, if the lesion (at first affecting the cortex and producing tactual insensibility) be carried deeper into the white substance, sensibility to pressure and sensibility to pain will become affected. It follows from the foregoing that, according to the depth of cerebral lesion made, we can abolish tactual sensation alone, or simultaneously diminish sensibility to pressure (and to pain). Such changes can be made to affect the hinder or fore extremity, or the face, at the side opposed to the lesion, by following the indications given by MM. Hitzig and Fritsch for the destruction of the so-called "motor", that is, the sensory centres of these organs. Professor Schiff holds, with Professor Hitzig, that there is no restoration of function possible in a centre which has been really destroyed. Apparent restoration occurs only where the destruction has been imperfect, or where the function had been temporarily abolished through the influence of an injury to a part in the vicinity.

Making use of the means indicated, anæsthesia of cerebral origin was produced in several dogs, and allowed to affect them to a greater or less extent. Some months were then permitted to elapse, in order that all transitory effects of the wound should have disappeared, after which experiments with the magnetising bobbin (to use Ampère's expression) were begun. The animal, having been placed on the table in an attitude of repose, the fore-limb (when that was experimented on) was passed through the cavity of the solenoid, which thus included the lower part of the humerus, whilst the protruding paw was free in order that the state of sensibility might be determined by irritating it. The mode of irritation adopted was of the mildest, consisting as it did in lightly stroking the hair with the finger-tips, avoiding pressure with the nail. Repeated tests showed that the anæsthesia remained unaltered whilst the animal was in this position, so long as the circuit was open. The effect of completing the circuit may best be understood by a short description of an experiment. A long-haired spaniel, which had been operated on some time before, had to all appearance completely recovered his normal condition, with the exception that, in walking over rough ground or going upstairs, he frequently leant his weight on the dorsal part of his toes, which Professor Schiff regards as an infallible sign of tactual insensibility. The presence of anæsthesia was carefully determined by other tests. When the animal was placed in position as described, if the dorsal hairs of the left (i.e., the sound) paw were slightly stirred by a quick downward touch, the paw was at once withdrawn, a thrill passed through the body, the hinder legs were momentarily extended, and that which was uppermost or free was kicked out. The muscles of the neck stiffened. The effect, like the irritation, was momentary. When the palmar surface was lightly and quickly touched, the result was less general, but the paw was drawn back, and the hinder leg kicked out. When the right (or anæsthetic) limb was irritated in the same manner, there was no movement whatever, neither in the paw nor elsewhere. Neither was there any movement when the hand was lightly closed and drawn over the included paw, though this had caused movement in the left limb and left side.

The circuit was closed. Five minutes afterwards, when the last-mentioned test was repeated, there resulted movements of extension and flexion of the articulation of the foot, and occasionally the toes spread out. Twenty minutes later, the excitability was found to have increased, for if the palmar surface of the paw were tickled by stirring the hairs, the foot was flexed; extension and flexion after contact had become more marked. The left extremity retained its sensibility. The acquired sensibility persisted during four or five hours, and even longer; when it disappeared, it could be reproduced in the same manner, sometimes in the course of the same day; but, generally speaking, on the following day. The result was subject to some modification when the experiment was performed on dogs of different temperaments; for in stance, in the case of a large dog, of less sensibility than the spaniel, recovery of tactual sensibility was only shown after pressure on the in:erdigital web, but here the posterior extremity showed signs of recovery as well, though the fore-limb alone had been placed within the solenoid

It is, however, unnecessary to enter into the details of the several experiments. The essential point is that, in all of them, recovery of tactual sensibility in the anresthetic region was cletected. In some animals there was less recovery, in others more; but in every one of the cases the anæsthesia gave way before the influence of the solenoid, in the cavity of which an isolated member was placed. These remarkable results, therefore, supply ample evidence of the correctness of my conclusions, and distinctly corroborate the report of Professor Charcot's Commission at La Salpêtrière.

\section{FATTY CHANGE (AND FAILURE) OF THE MUSCULAR WALL OF THE GUT, AS A DIRECT AND INDIRECT CAUSE OF INTESTINAL OBSTRUCTION AND DEATH.}

By F UR NEAUX JORDAN, F.R.C.S.,

Surgeon to the Queen's Hospital, Professor of Surgery at Queen's College, Birmingham, Consulting Surgeon to the Women's and the West Bromwich Hospitals.

For several years past, I have from time to time seen cases in which, with, perhaps, no premonitory symptoms, continuous vomiting and tympany, lasting one, two, or more days, have been followed by death. While these symptoms appeared in some cases to come on spontaneously, in others, and I think more frequently, they followed some abdominal or pelvic operation. The cases, as a rule, happened in fat persons, in persons with large abdomens, in persons with signs of degeneration in various organs and with a history of habits which naturally lead to visceral changes. Examination of the bodies disclosed great internal accumulations of fat, and occasionally indications of visceral degeneration, but, curiously, no obvious or recognised cause of intestinal obstruction. In all the cases, the intestinal canal was greatly loaded with fat, and presented a strikingly yellow appearance; in some cases, indeed, seemed to be simply a tube of fat. In one case, the microscope conclusively showed that the unstriped muscular fibres of the bowel were converted into fat. In observing and reflecting on these cases, of some of which I shall speak later, I have arrived at the following conclusions. I. The smooth muscular fibres of the bowel are subject to fatty degeneration, which may become more or less complete; and that, consequently, they may, and do in given cases, wholly cease to contract.

2. This fatty change of the essential element of the gut-wall, when it ends in complete cessation of contractility, causes death by intestinal obstruction. Fatty failure of the intestines being in some cases extensive in area and reaching high up towards the stomach, the ensuing obstruction is acute, the vomiting incessant, and death early. In other cases, there may be less complete, or more limited, or irregularly distributed fatty change; and there will follow a slower or more fitful stream of symptoms and a later death.

3. Fatty transformation in the gut is more likely to appear (though perhaps not exclusively) in fat, especially very fat persons; in those who, from habits or natural tendency, are liable to have fatty degeneration of other organs, especially of the heart. Death in heartcases is quick and direct; in intestinal-cases, slower and more indirect, but nevertheless very certain.

4. As premonitory syncope or exhaustion may happen from time to time before death from heart-fattiness, so "attacks" of obstruction may run before final obstruction from intestinal fattiness.

5. Failure of the bowel is helped on by continued flatulent distension, however it arises; the altered muscular fibres being so injured by overstretching that they never regain their functional contractility. Herein may be traced a likeness to atony of the bladder, where, it is well known, long-continued distension is in certain cases followed by entire loss of contractility; and it is not unlikely that fatty conversion of the muscular wall of the bladder is the basis of certain obscure cases of retention and cystitis coming on after middle age. It is conceivable that healthy gut may become the subject of fatal atony from longcontinued stretching; but some, however slight, fatty chang would, greatly favour such a result.

6. In a limited number of cases, death is due directly to failure of intestinal action, and may come without obvious exciting cause. The muscular fibre is now no longer muscular. In a larger number of cases, death comes more indirectly from some immediate shock to the abdominal organs. In strangulated hernia, when fatty bowel is present the, blown-out tube never again contracts. The vomiting continues, or returns, and death follows, notwithstanding that reduction has been easy and complete, and that there is no inflammation, or gangrene, or other cause of death. All injuries and operations in persons with failing gut are liable to be followed by vomiting, which ceases only with death. Especially is this so in operations on the abdominal or pelvic organs. Herniotomy and lithotomy are now and then followed by fatal vomiting, and subsequent search brings nothing to light; no injury to the peritoneum, no hæmorrhage, no inflammation, no other lesion; nothing but a hugely clistenc?ed bowel. 